Rev Biomed 2005; 16:79-85.

\title{
Sucesión de larvas de muscoideos durante la degradación cadavérica en un bosque premontano húmedo tropical.
}

\section{Artículo Original}

Olger Calderón-Arguedas ${ }^{1}$, Adriana Troyo $^{1,2}$, Mayra E. Solano ${ }^{1}$.

${ }^{1}$ Centro de Investigación en Enfermedades Tropicales (CIET), Departamento de Parasitología, Facultad de Microbiología, Universidad de Costa Rica, San José, Costa Rica, ${ }^{2}$ Department of Epidemiology and Public Health, University of Miami, FL, USA.

\section{RESUMEN.}

INTRODUCCIÓN. La sucesión de larvas de muscoideos, reconocida herramienta en las investigaciones médico legales, fue estudiada como un criterio en la estimación del intervalo post mortem (IPM) en un modelo experimental con conejos.

MATERIAL Y MÉTODOS. Durante cuatro ciclos trimestrales que iniciaron en abril, junio, octubre del 2002 y enero del 2003, se sacrificaron por dislocación cervical 3 conejos de la cepa New Zealand $(1,8 \mathrm{~kg})$ los cuales fueron expuestos en un ambiente de bosque premontano húmedo tropical. A partir de los cadáveres, tres veces a la semana, se colectaron formas larvales de muscoideos las cuales fueron fijadas en etanol al 70\% y aclaradas en lactofenol para su posterior identificación por microscopia de luz. Adicionalmente se realizó la determinación de las temperaturas del suelo, de la interfase cadáver-suelo y de la masa larval.

RESULTADOS. El modelo no permitió observar una variación marcada en la entomofauna asociada con las fases de descomposición cadavérica, por lo que la información obtenida orienta acerca del período de muerte, pero no precisa el tiempo de muerte. Los califóridos fueron buenos indicadores de la fase coagulativa. De estos, las especies Hemilucilia segmentaria y Lucilia eximia fueron evidenciadas en todos los ciclos de muestreo. Synthesiomyia nudiseta (Diptera: Muscidae) fue una especie bastante frecuente en la fase de descomposición avanzada. Ejemplares de las familias Fannidae, Sepsidae, Micropezidae y Drosophilidae, fueron colectados en los últimos días del período de observación (fases de descomposición avanzada y seca). En relación con las temperaturas, la temperatura de la masa larval fue más alta durante la fase de descomposición activa en todos los ciclos. Durante los ciclos I y IV, correspondientes a los meses secos, la temperatura de la interfase cadáver suelo fue también elevada durante la fase de descomposición activa.

DISCUSIÓN. Aunque el análisis de formas larvales de muscoideos no constituye un método que garantice precisión en las estimaciones del IPM, la información derivada da una idea de la temporalidad y de la

Solicitud de sobretiros: Dr. Olger Calderón-Arguedas. Departamento de Parasitología, Facultad de Microbiología, Universidad de Costa Rica,San José, Costa Rica. E-mail: olgerc@cariari.ucr.ac.cr Recibido el 8/Marzo/2005. Aceptado para publicación el 23/Mayo/2005. 
O Calderón-Arguedas, A Troyo, ME Solano.

localización geográfica que pueden ser claves para la interpretación de los análisis forenses.

(Rev Biomed 2005; 16:79-85)

Palabras clave: Entomología forense - Hemilucilia segmentaria- Synthesiomyia nudiseta Calliphoridae-Muscidae-Costa Rica

\section{SUMMARY.}

Succession of muscoid larvae during cadaveric decay in a tropical premontane humid forest. INTRODUCTION: The succession of muscoid larvae, a common tool in medical-legal investigations, was studied as a criterion for the estimation of the Post Mortem Interval (PMI) in an experimental model with rabbits.

MATERIAL AND METHODS: During four trimestral cycles that began in April, June, October of 2002, and January of 2003, three New Zealand rabbits $(1,8 \mathrm{~kg})$ were euthanasied by cervical dislocation and exposed in a tropical premontane humid forest. Three times a week, muscoid larvae were collected from each corpse. They were fixed in ethanol $70 \%$, cleared in lactophenol, and mounted in Hoyer's medium for their observation by light microscopy. In addition, temperatures of the soil, soil-corpse interphase, and maggot mass were determined.

RESULTS: Even though the model did not show great variation in the entomofauna associated to each decomposition phase, the generated information could approximate a time period since death, but not the exact time of the death. The calliphorids were good indicators for the bloating phase: Hemilucilia segmentaria and Lucilia eximia were evidenced in all the sampling cycles. In the active decomposition phase, Synthesiomyia nudiseta (Diptera: Muscidae) was a highly frequent species. Larvae of Fannidae, Sepsidae, Micropezidae and Drosophilidae were collected in the last days of the observation period (advanced decomposition and dry phases). Regarding the temperatures, the maggot-mass temperature was higher during active decomposition in all the cycles. During cycles I and IV, corresponding to the dry months, the temperature of the soil-corpse interphase was also higher in the active decomposition phase. DISCUSSION: Although analysis of larval types are not methods that precisely estimate the PMI, the information derived gives an idea of temporality and geographic location, which can be key aspects in the interpretation of the forensic analysis.

(Rev Biomed 2005; 16:79-85)

Key words: Forensic Entomology - Hemilucilia segmentaria- Synthesiomyia nudisetaCalliphoridae-Muscidae - Costa Rica

\section{INTRODUCCIÓN.}

La degradación cadavérica cursa por una serie de fases las cuales, aunque pueden variar dependiendo de las condiciones medio ambientales y el tamaño de los cuerpos, se manifiestan de manera más o menos constante. Anderson y VanLaerhoven (1), mediante un modelo experimental realizado en cerdos pudieron reconocer las siguientes fases: fresca (primeras 24 horas), coagulativa $\left(2^{\circ}\right.$ a $10^{\circ}$ día), descomposición activa $\left(11^{\circ}\right.$ a $16^{\circ}$ día), descomposición avanzada $\left(17^{\circ}\right.$ a $42^{\circ}$ día) y finalmente la fase seca (luego del $43^{\circ}$ día).

En cada una de estas fases y como resultado de los cambios físico-químicos que tienen lugar, se da la colonización por parte de diferentes grupos de insectos necrófagos así como de sus respectivos predadores $(2,3)$.

El análisis de la entomofauna asociada con cadáveres ha sido un criterio utilizado en las investigaciones forenses $(3,4)$. Las especies involucradas podrían revelar datos importantes acerca de la ubicación geográfica de la escena de muerte y su composición cualitativa podría brindar criterios para el cálculo del intervalo post mortem (IPM), el cual consiste en la estimación de los tiempos probables máximo y mínimo desde el deceso hasta el descubrimiento del respectivo cadáver $(3,5)$.

Dada la diversidad biológica relativa a número de especies que tiene lugar en Costa Rica, se planteó la realización de un modelo experimental en conejos, con la idea de identificar especies y grupos taxonómicos de larvas de muscoideos que pudiesen

\section{Revista Biomédica}




\section{Sucesión de larvas de muscoideos durante la degradación cadavérica.}

servir como indicadores forenses en el área de estudio.

\section{MATERIALESY MÉTODOS.}

Como parte de un estudio en el que se han valorado diversos tópicos de entomología forense, se evaluó la composición cualitativa de larvas de muscoideos en muestras colectadas a partir de cadáveres como un criterio para estimar el tiempo de muerte. En el presente modelo se realizaron observaciones a partir de carcasas de conejo durante cuatro ciclos de observación (I-IV) que iniciaron en los meses de abril, julio y octubre del 2002 y enero del 2003. En cada uno de los ciclos, tres conejos de la cepa New Zealand $(1,8 \mathrm{~kg})$ fueron sacrificados por dislocación cervical, siguiendo los procedimientos éticos respectivos. Los cadáveres fueron expuestos a la intemperie a una distancia mínima de 20 metros entre ellos, en un ambiente de bosque premontano húmedo tropical representado por la reserva "Leonel Oviedo" en la Ciudad Universitaria "Rodrigo Facio" de la Universidad de Costa Rica. La vegetación dominante de esta reserva está constituida por Justicia sp. (Acanthacea), Cinnamomun cinnamonifolia (Laureacea) y Cupania glabra (Sapindacea) (6). Los promedios de temperatura, humedad y precipitación para el lugar son de $19,3^{\circ} \mathrm{C}, 79,8 \%$ y $19,5 \mathrm{~mm}$ para la estación seca (diciembre-abril) y $20,2^{\circ} \mathrm{C}, 85,1$ y $245,4 \mathrm{~mm}$ para la lluviosa (mayo-noviembre).

Para evitar la acción de posibles depredadores, cada cadáver fue depositado en una jaula de cedazo calibre 19 (apertura de 2,5 x 2,5 cm) cuyas medidas fueron $80 \times 30 \times 25 \mathrm{~cm}$. Las jaulas fueron sujetadas al suelo circundante con la ayuda de ganchos especializados. Los cadáveres fueron estudiados trisemanalmente desde el día 3 hasta el 80 postexposición. En cada muestreo se recolectaron, una a una, larvas de mosca por un período de cinco minutos. Dichas larvas fueron colocadas en viales con etanol al $70 \%$ para su conservación y transporte al laboratorio. El análisis de las muestras implicó una observación al microscopio estereoscópico (4X) para la identificación preliminar de los diferentes morfotipos larvales presentes. Ejemplares representativos de los mismos fueron aclarados en lactofenol y montados en medio
Hoyer para su respectiva identificación microscópica, la cual se basó en claves dicotómicas especializadas $(7,8)$.

Adicionalmente, se realizó la determinación de las temperaturas: ambiental, de la interfase cadáver/ suelo y de la masa larval. Dicha determinación, así como la colecta de material entomológico, se llevó a cabo entre las 9:00 y las 10:00 AM en los días de observación. La categorización de las fases de degradación cadavérica fueron hechas con base en los criterios descritos por Anderson y VanLaerhoven (1) (cuadro 1).

Considerando que la fase fresca transcurre durante las primeras 24 horas, y es poco frecuente la colecta de larvas de muscoideos en este período, la misma no fue considerada para la presentación de resultados.

\section{RESULTADOS.}

La descomposición cadavérica observada en el modelo permitió identificar las fases propias del proceso (cuadro 2). Con respecto a la fase coagulativa tanto en el ciclo I y II, las larvas identificadas fueron mayoritariamente de las familias Calliphoridae y Sarcophagidae, aunque fóridos y múscidos también fueron evidenciados. Cuatro especies de califóridos fueron identificadas: Hemilucilia segmentaria, Lucilia eximia, Chrysomya megacephala y Ch. rufifacies. $H$. segmentaria y $L$. eximia fueron observadas en los cuatro ciclos de muestreo (cuadro 2, figura 1). En la fase de descomposición activa se mantuvieron los grupos mencionados anteriormente pero el hallazgo de Synthesiomyia nudiseta de forma dominante junto con las larvas de Phoridae fue generalizado en los cuatro ciclos (cuadro 2, figura 1). En la fase de descomposición avanzada fue posible identificar algunas otras familias no documentadas preliminarmente. Dentro de ellas figuran Drosophilidae, Fannidae, Micropezidae y Sepsidae. Sólo en el ciclo III no fue posible evidenciar representantes de estas familias (cuadro 2).

En la fase seca, con la excepción del tercer ciclo, donde se documentó la recolección de ejemplares 
O Calderón-Arguedas, A Troyo, ME Solano.

\section{Cuadro 1}

Condensación de las características correspondientes a las fases de descomposición cadavérica de acuerdo a Anderson y VanLaerhoven (1).
Fase
Características

Fresca

Sin olor. Enfriamiento corporal. No hay cambios morfológicos notables.

Coagulativa

Ligera inflamación del abdomen. Olor putrefacto leve. Pérdida de peso. Piel íntegra.

Descomposición Desinflamiento y ruptura de la dermis. Masa muscular y piel presentes pero parcialmente activa degradadas. Olor fuerte. Carcasa con apariencia húmeda. Reducción al 50\% de la masa muscular.

Descomposición Ruptura evidente de la piel. Musculatura parcial o totalmente degradada. Disminución avanzada ulterior de la masa muscular y piel. Caída en la intensidad de olor.

Seca/restos $\quad$ Presencia de huesos, cartílago y piel secos. Masa muscular mínima o nula, olor leve o nulo.

Cuadro 2

Morfotipos larvales observados en las carcasas durante los cuatro ciclos de observación.

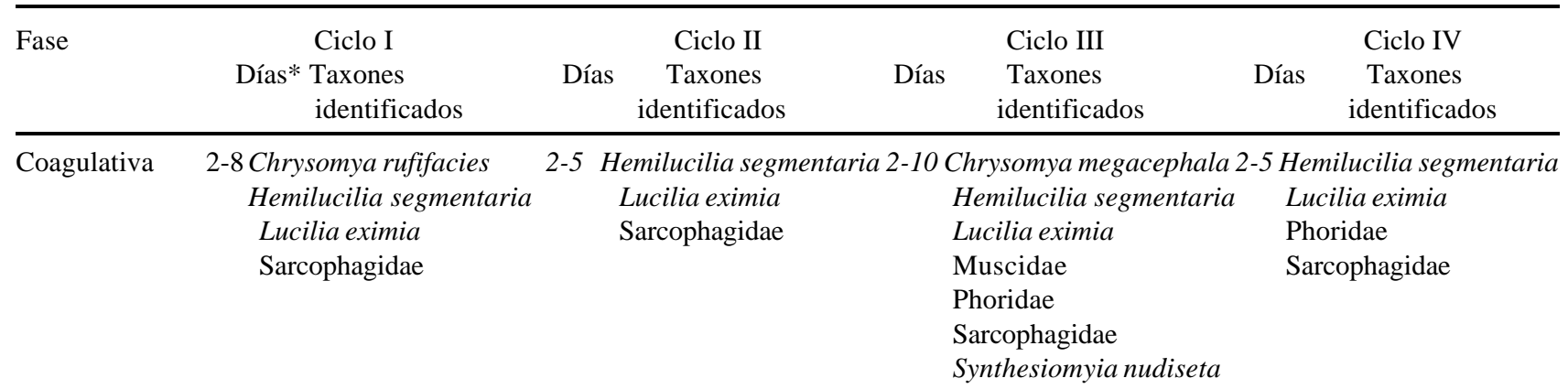

Descomposición 9-33 Chrysomya rufifacies 6-10 Hemilucilia segmentaria 11-27 Chrysomya megacephala 6-15 Chrysomya megacephala Activa

Hemilucilia segmentaria

Lucilia eximia

Ophyra sp.

Phoridae

Sarcophagidae

Synthesiomyia nudiseta

Descomposición 34-75 Drosophilidae avanzada

Fannia scalaris

Micropezidae

Phoridae

Sepsidae

Synthesiomyia nudiseta

Seca
Lucilia eximia

Ophyra sp.

Phoridae

Sarcophagidae

Synthesiomyia nudiseta
Hemilucilia segmentaria

Muscidae

Phoridae

Sarcophagidae

Synthesiomyia nudiseta
Chrysomya rufifacies

Hemilucilia segmentaria

Lucilia eximia

Muscidae

Phoridae

Sarcophagidae

Synthesiomyia nudiseta

11-24 Fannia scalaris 28-30 Chrysomya megacephala 16-20 Chrysomya rufifacies

Hemilucilia segmentaria

Phoridae

Sarcophagidae

Sepsidae

Synthesiomyia nudiseta
Fannia scalaris

Phoridae

Sarcophagidae

Synthesiomyia nudiseta
Hemilucilia segmentaria Ophyra sp.

Phoridae

Sarcophagidae

Synthesiomyia nudiseta

\begin{tabular}{|c|c|c|c|c|}
\hline Seca & $\begin{array}{l}\text { 76-80 Phoridae } \\
\text { Synthesiomyia nudiseta }\end{array}$ & $\begin{array}{l}\text { 25-80 Drosophilidae } \\
\text { Hemilucilia segmentaria } \\
\text { Phoridae } 1 \\
\text { Phoridae } 2\end{array}$ & $\begin{array}{l}\text { 33-80 Chrysomya megacephala } \\
\text { Hemilucilia segmentaria } \\
\text { Phoridae } \\
\text { Piophilidae } \\
\text { Sarcophagidae } \\
\text { Synthesiomyia nudiseta }\end{array}$ & $\begin{array}{l}\text { 21-80 } \\
\text { Ophyscidae } \\
\text { Phoridae } \mathrm{sp.} \\
\text { Sarcophagidae } \\
\text { Synthesiomyia nudiseta }\end{array}$ \\
\hline
\end{tabular}

* Días post-exposición

Revista Biomédica 
Sucesión de larvas de muscoideos durante la degradación cadavérica.

pertenecientes a la familia Piophilidae, no se pudieron observar grupos diferentes a los ya citados. En tres de los ciclos la colecta de califóridos fue nula en esta fase.

La determinación de las temperaturas permitió advertir un aumento marcado en la temperatura de la masa larval en todos los ciclos durante la fase de descomposición activa. También en esta fase se pudo evidenciar un aumento en la temperatura de la interfase cadáver-suelo en los ciclos I y IV que corresponden a los meses de menor precipitación (figura 2).

En el tercer ciclo de observación la degradación cadavérica total tuvo lugar alrededor del día 35 , por
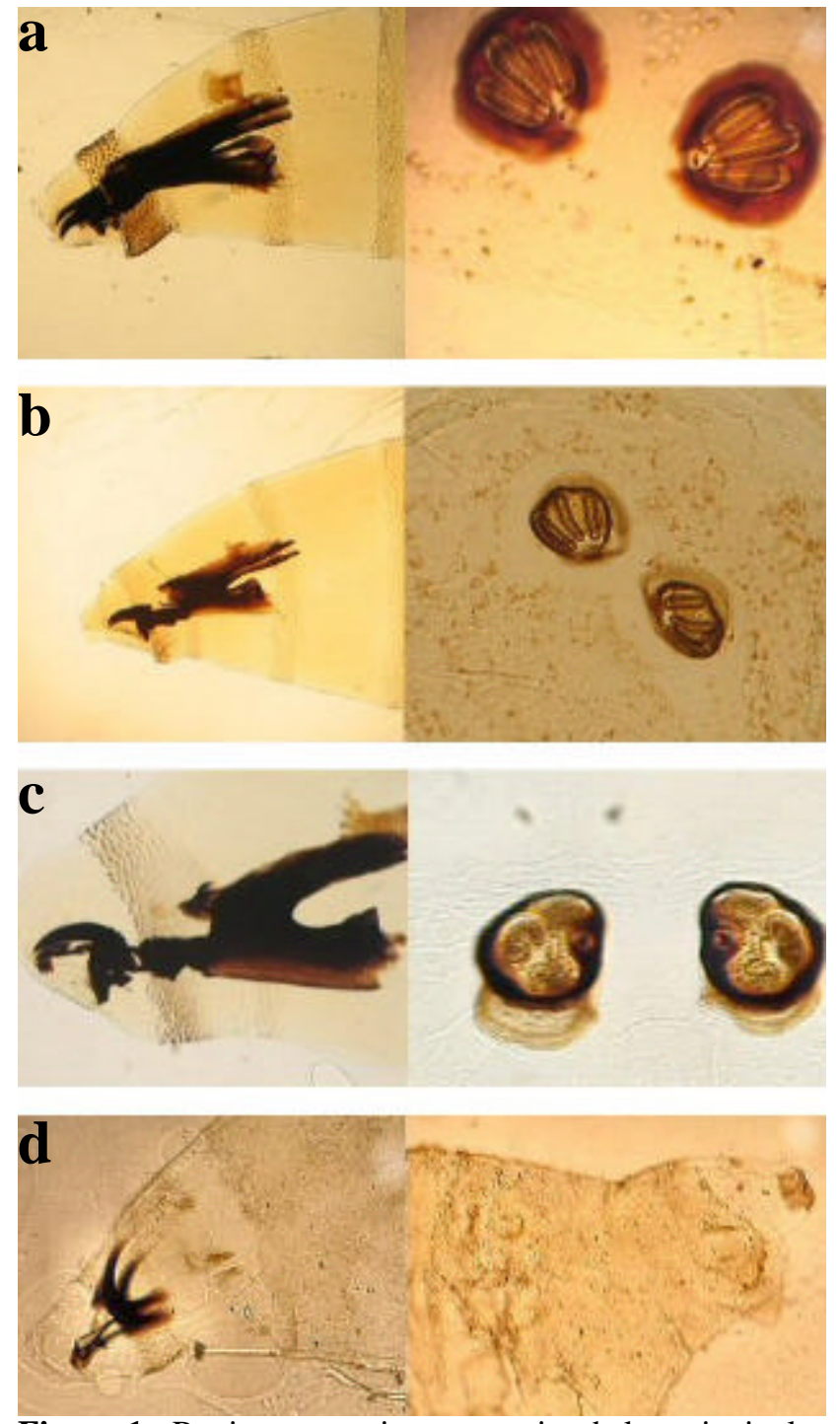

Figura 1.- Regiones anterior y posterior de los principales morfotipos encontrados. a: H. segmentaria, b: L. eximia, c: S. nudiseta, d: Phoridae lo que la determinación de temperaturas fue llevada a cabo hasta este día (figura 2).

\section{DISCUSIÓN.}

Algunas investigaciones sobre entomología forense han encontrado una relación directa entre la descomposición cadavérica y la entomofauna asociada (1). No obstante, el experimento realizado, enmarcado en un país tropical como Costa Rica, no permitió la identificación de variaciones abruptas en la composición cualitativa de las formas larvales presentes en las muestras que pudiesen diferenciar marcadamente las fases de descomposición cadavérica. El análisis realizado permitió orientar acerca del período de muerte, pero la imposibilidad de darle connotación cuantitativa a las observaciones dificultó el establecimiento de conclusiones definitivas. En relación con la estimación del IPM con base en muestras entomológicas, Catts (5) realizó un análisis de las dificultades que pueden cursar en este tipo de investigaciones, algunas de las cuales podrían estar afectando los resultados obtenidos. Dentro de éstas, se deben destacar las condiciones a las que ha sido sometido el cadáver (localización, temperatura, humedad y tratamiento post mortem). En este sentido, las características de los cuerpos en relación con masa corporal, así como las circunstancias de muerte, pueden modular la colecta entomológica. Por ejemplo, si los cadáveres están cubiertos o quemados, la sucesión podría darse parcialmente o no darse del todo. Por otro lado, la ingestión de drogas o sustancias químicas por parte del occiso podrían constituir agentes selectivos que limiten la oviposición de los insectos necrófagos.

Considerando que la colonización larval no se da uniformemente, sino que ésta inicia con predilección por los orificios naturales o sitios que presentan algún tipo de exudación, se plantea el problema de cómo y dónde se debe muestrear, aspectos que usualmente no son abordados en las investigaciones de este tipo. Otro problema asociado es de carácter práctico, ya que los personeros encargados de la colecta usualmente no tienen formación entomológica, por lo que la 


\section{O Calderón-Arguedas, A Troyo, ME Solano.}

representatividad de la muestra podría no ser la óptima (5).

En el presente modelo y tal como se ha documentado previamente (4), los califóridos se comportaron como buenos indicadores de la fase coagulativa. Estas moscas suelen localizar los cadáveres en un período que puede ir desde minutos hasta horas luego de la muerte y de esta manera iniciar sus procesos de oviposición y colonización (4). En este sentido, H. segmentaria y L. eximia (figura 1) se presentaron como especies dominantes en todos los ciclos de observación, razón por la cual se podría especular que sus poblaciones persisten durante todo el año. No obstante, la particularidades morfológicas

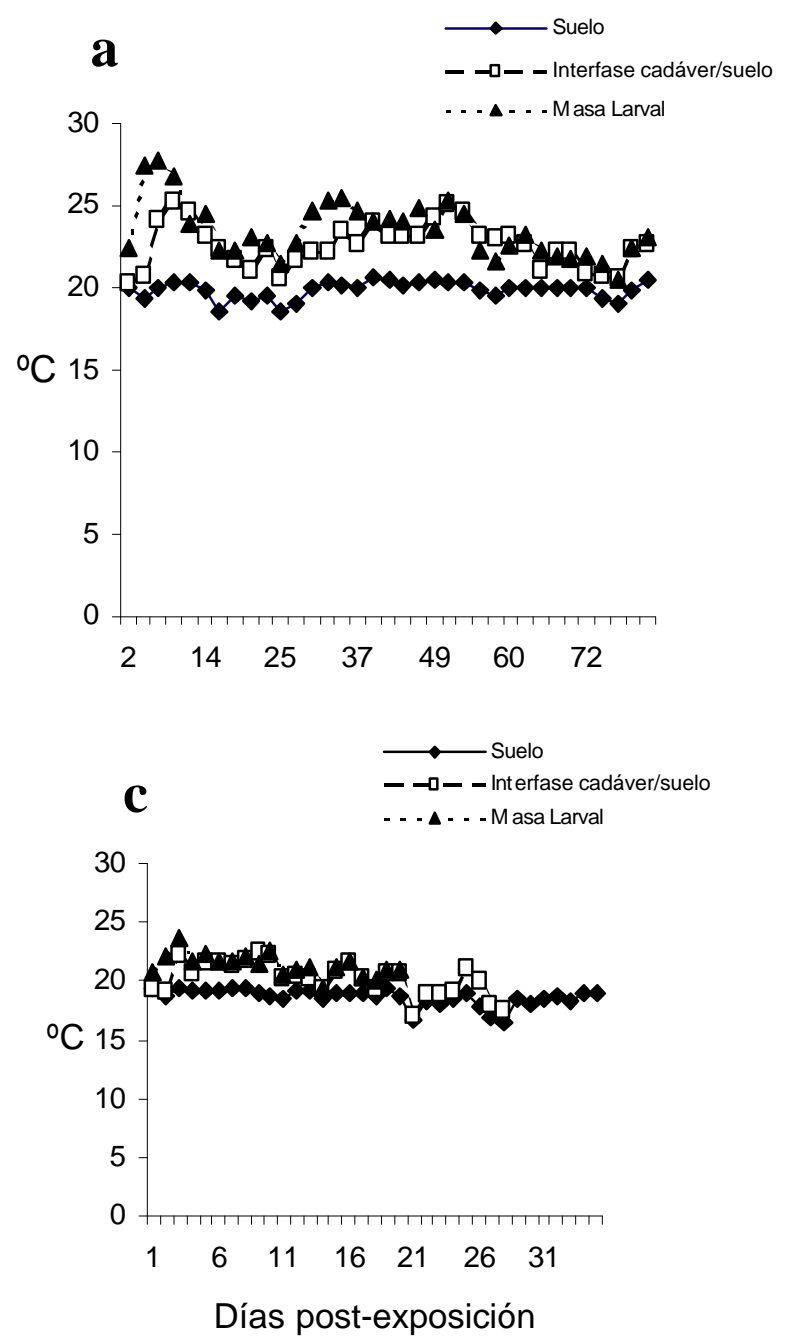

de $H$. segmentaria, que la hacen fácilmente identificable, la perfilan como un adecuado indicador para esta etapa. Aunque los sarcofágidos fueron igualmente frecuentes en esta fase, la poca disponibilidad de información taxonómica plantea dificultades en su utilización como indicadores forenses.

En la fase de descomposición activa, llama la atención la recuperación generalizada de $S$. nudiseta. Este díptero ha sido encontrado previamente en cadáveres expuestos en Costa Rica (9). Su alta frecuencia de recuperación permite sugerir a esta especie como un indicador para esta fase.

Aunque larvas de la familia Phoridae
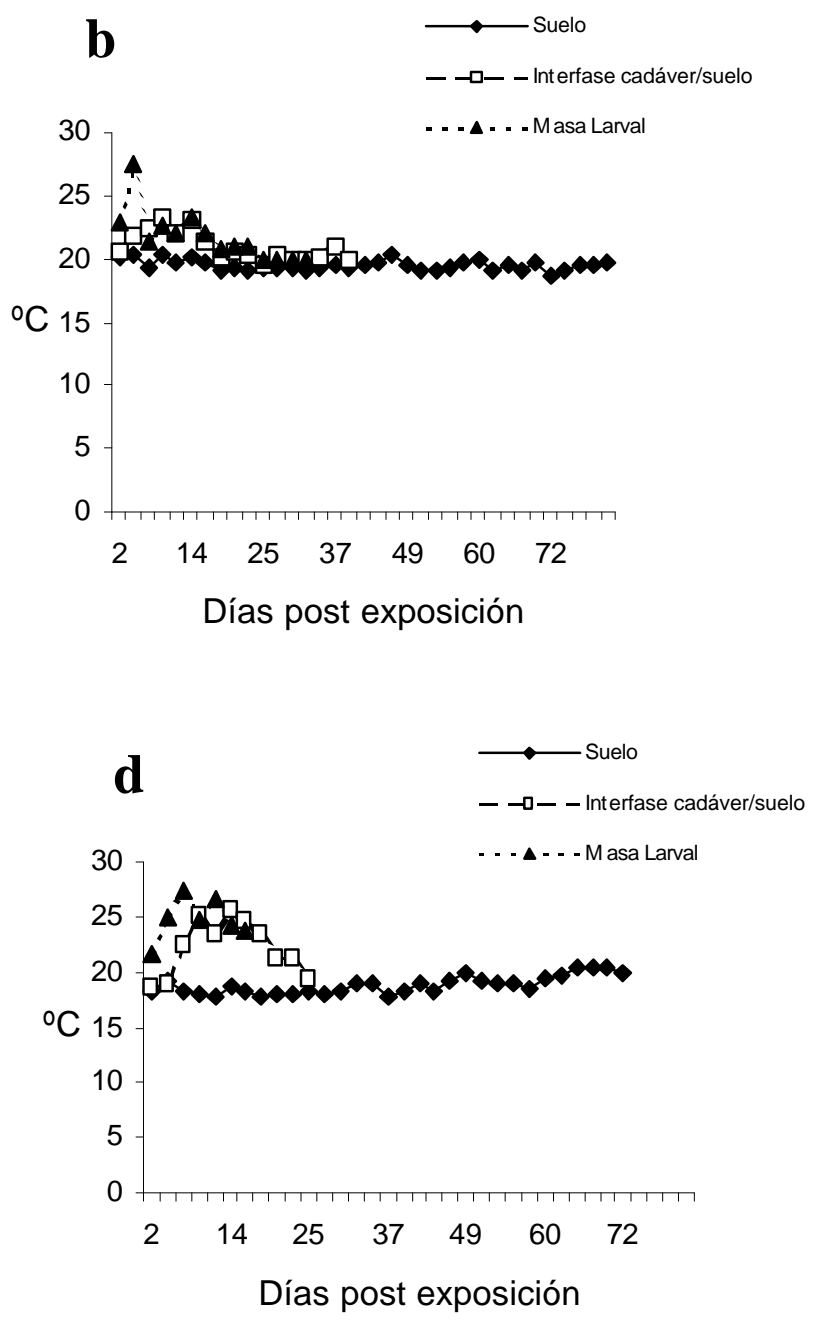

Figura 2.- Promedios de las temperaturas monitoreadas en los cuatro ciclos de observación. a: Ciclo I (abril-junio/2002), b: Ciclo II (julio-septiembre/2002), c: Ciclo III (octubre-diciembre/2002), d: Ciclo IV (enero-marzo/2003). 


\section{Sucesión de larvas de muscoideos durante la degradación cadavérica.}

generalmente aparecen cuando inician los procesos de saponificación cadavérica (2), también se encontraron de forma generalizada en el presente estudio (figura 1). Estas continuaron siendo observadas hasta el final del experimento sin permitir discriminar las fases posteriores.

En la descomposición avanzada tuvo lugar la aparición de algunos grupos, que aunque no son generalizables a todos los ciclos, podrían servir para la identificación de esta fase. Entre estos grupos figuran las familias Fannidae, Sepsidae Micropezidae y Drosophilidae. Tal y como se aprecia en el cuadro 2 , el modelo no permitió una diferenciación marcada entre la fase de descomposición avanzada y la fase seca. Lo único pertinente fue una discreta disminución en el número total de taxones recuperados.

El análisis de las temperaturas permitió observar valores relativamente constates durante todo el período de observación, destacándose un discreto aumento en la temperatura de la masa larval durante la fase de descomposición activa en todos los ciclos (figura 2). Esto difiere de otros resultados donde se han documentado incrementos significativos en esta temperatura que ha llegado hasta $\operatorname{los} 40^{\circ} \mathrm{C}(5)$. Lo anterior podría estar relacionado con el tipo de carcasa utilizado en el modelo, el cual por su tamaño podría facilitar la pérdida de calor. Este aspecto podría afectar el número y tipo de larvas recuperadas ya que se podría dar la ralentización del desarrollo en comparación a lo que podría ocurrir en carcasas grandes. También se advirtió un discreto aumento de las temperatura de la interfase cadáver-suelo en los ciclos I y IV los cuales corresponden a los meses de menor precipitación (figura 2). Este aumento podría estar relacionado con una descomposición cadavérica más lenta la cual permite la permanencia de artrópodos y microorganismos de forma más prolongada. Estos contribuyen a la generación de calor como resultado de sus actividades metabólicas aunado a que la disipación de calor es menor cuando la humedad circundante es baja $(3,5)$.

En términos generales, se puede decir que los análisis de formas larvales de muscoideos no constituyen un método que garantice precisión y exactitud en las estimaciones del IPM. Sin embargo, la información derivada de los mismas da una idea de la temporalidad y de la localización geográfica que pueden ser claves para la interpretación de los análisis forenses.

\section{AGRADECIMIENTOS.}

Los autores desean externar su agradecimiento a Iván Coronado, Ronald Mora, Jacqueline Moya, María Soledad Faba y Adrián Bonilla por su labor operativa; a Francisco Di Stéfano Ph. D., Escuela de Biología en la Universidad de Costa Rica (UCR), por su anuencia en la utilización de la Reserva "Leonel Oviedo" y a la Vicerrectoría de Investigación (UCR) por su apoyo financiero al proyecto 803-A2-041.

\section{REFERENCIAS.}

1.- Anderson GS, VanLaerhoven SL. Initial studies on insect succession on carrion in Southwestern British Columbia. J Forensic Sci 1996; 41: 617-25.

2.- Turcheto M, Vanin S. L' Entomologia forense e la globalizzazione. Parassitologia 2004; 46:187-90.

3.- Catts EP, Goff ML. Forensic entomology in criminal investigations. Annu Rev Entomol 1992; 253-72.

4.- Hall M, Donovan S. Forensic entomology: what can maggots tell us about murders? Biologist 2001; 48: 249-53.

5.- Catts EP. Problems in estimating the postmortem interval in death investigations. J Agric Entomol 1992; 9: 245-55.

6.- Di Stéfano JF, Nielsen V, Hoomans J, Fournier LA. Regeneración de la vegetación arbórea en una pequeña reserva forestal urbana del nivel premontano húmedo, Costa Rica. Rev Biol Trop 1996; 44: 275-80.

7.- Vargas-Fonseca J. Distribución y morfología de adultos e inmaduros de moscas califóridas (Diptera) de importancia forense en Costa Rica (Tesis). Facultad de Ciencias Básicas. Escuela de Biología. Universidad de Costa Rica; 1999.

8.- James, MT. The flies that cause myiasis in man, United States Department of Agriculture, Pub 63. Washington D. C. USDA, 1947. p. 1-175.

9.- Jirón LF, Vargas LG, Vargas-Alvarado E. Four muscoid flies (Sarcophagidae and Muscidae) associated with human cadavers in Costa Rica. Brenesia 1983; 21: 3-5. 